ORIGINAL

\title{
Lower axillary dissection in breast cancer surgery may be candidate for cases with early breast cancer
}

\author{
Masatsugu Takehisa, ${ }^{1,3}$ Taeko Nagao', Mitsuteru Yoshida', Toshiyuki Hirose ${ }^{1}$, \\ Aiichiro Kajikawa', Mitsunori Sasa ${ }^{2}$, and Akira Tangoku ${ }^{3}$ \\ ${ }^{1}$ Department of Surgery, Higashi Tokushima National Hospital, National Hospital Organization, Tokushima, \\ Japan ; ${ }^{2}$ Tokushima Breast-Care Clinic, Tokushima, Japan; and ${ }^{3}$ Department of Oncological and \\ Regenerative Surgery, Institute of Health Biosciences, The University of Tokushima Graduate School, \\ Tokushima, Japan
}

\begin{abstract}
Lower axillary lymph node dissection (lower parts of both the level I and II elements below the second intracostobrachial nerve) and level I and II lymph node dissection were performed on breast cancer patients $(n=54)$, and the results with the two methods were compared in terms of the status of detected lymph node metastases. For Stage I, N0 cases, the results for pathological classification lymph node metastases $(\mathrm{pN})$ were in agreement between the two dissection methods. And, the occurrence of operated arm swelling wasn't recognized when a side effect was examined with the case $(n=28)$ that only lower axillary dissection was carried out in case of an operation for breast cancer. Accordingly, it was surmised that lower axillary dissection provides accurate $\mathrm{pN}$ information for Stage $\mathrm{I}$, NO cases. These results indicate that lower axillary dissection has the potential to become an effective, standard surgical procedure for breast cancer patients whose preoperative disease stage is Stage $I$.
\end{abstract}

J. Med. Invest. 52 : 74-79, February, 2005

Keywords :early breast cancer, axillary dissection, sentinel node biopsy

\section{INTRODUCTION}

The objectives of axillary lymph node dissection in breast cancer surgery are to provide reliable information regarding the number of pathological lymph node metastases $(\mathrm{pN})$ and to thereby prevent axillary lymph node recurrence. At present, level I and II dissection is recommended as the standard technique for axillary lymph node dissection.

However, this dissection is associated with such complications as swelling of the arm on the dissected side. As a result, sentinel lymph node biopsy is currently

Received for publication November 8, 2004 ; accepted January 5, 2005.

Address correspondence and reprint requests to Masatsugu Takehisa, Department of Surgery, Higashi Tokushima National Hospital, National Hospital Organization, Tokushima 779-0193, Japan and Fax: +81-88-672-3809. undergoing clinical trials as a new technique at various institutions. However, radioisotopes (RI) must be used in combination with sentinel lymph node biopsy in order to elevate the accuracy rate. This means that sentinel lymph node biopsy can be applied only at hospitals which are equipped to use RI techniques.

In view of this situation, the present authors conceived of and have carried out lower axillary dissection in breast cancer surgery. As described herein, we have found that this technique results in fewer complications while also providing accurate prognostic information.

\section{SUBJECTS AND METHODS}

\section{(STUDY 1)}

The 28 examples in the person who carried out the primary breast cancer operation which entailed axillary 


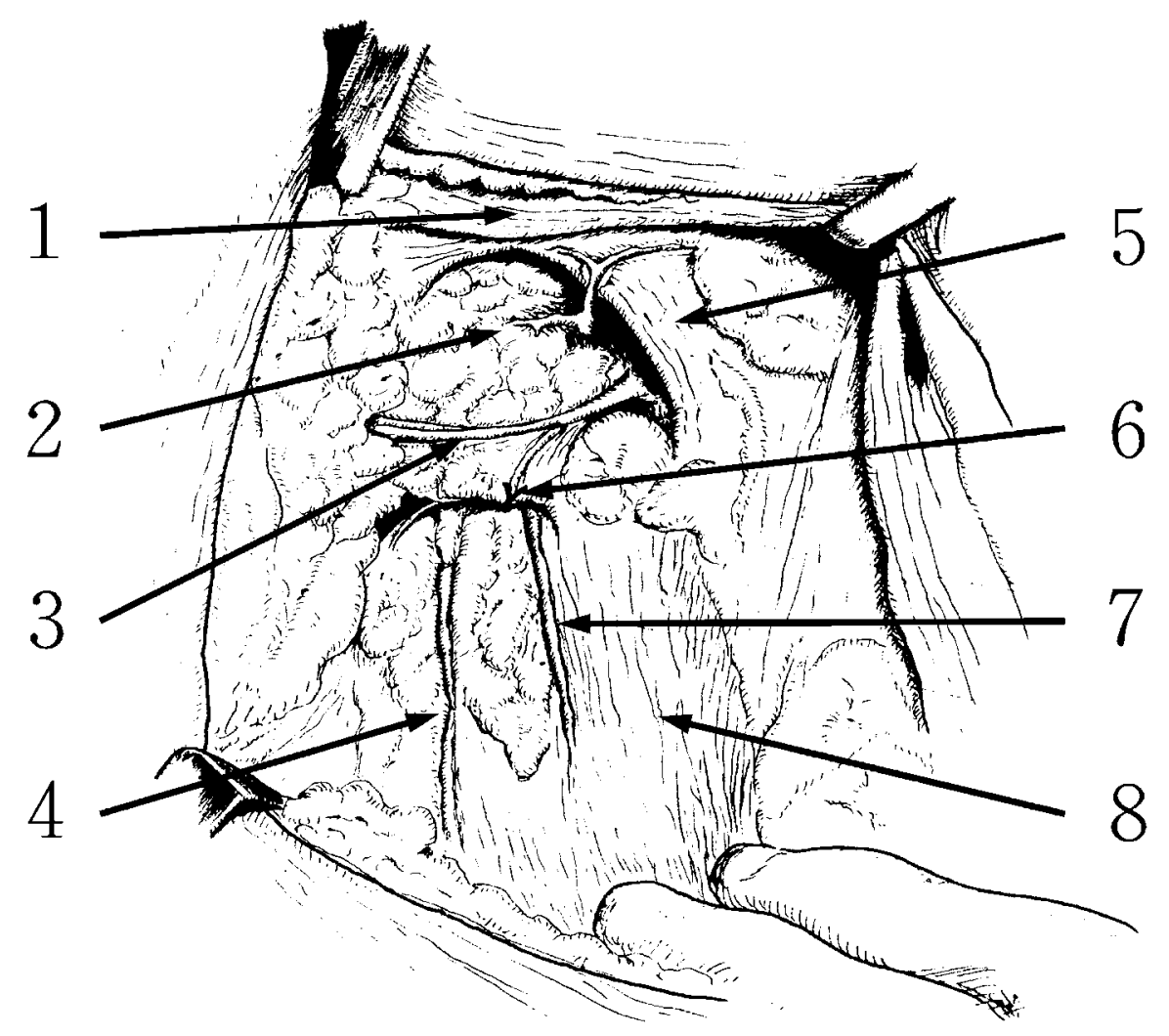

Fig. 1. Diagram of the axillary region after lower axillary dissection (right side) 1. Larger pectoral muscle ; 2 . Axillary vein ; 3 . Intercostobrachial nerve ;

4. Thoracodorsal nerve ; 5 . Smaller pectoral muscle ; 6 . Lateral thoracic artery stump ;

7. Long thoracic nerve ; 8 . Serratus anterior muscle

lymphnode dissection in the east Tokushima hospital from 1997 by January, 2001 were made the target. It is because the reason why lower axillary dissection was carried out was the advanced age of seventy years old and over 12 cases, a preoperative stage was Stage 0 -I 15 cases, a dialysis patient 2 cases. Our technique for lower axillary dissection is as follows.

The second intercostobrachial nerve passes out of the thoracic cavity and runs across the axillary region in the direction of the upper arm. Using this as the boundary, the axillary lymph nodes are divided into upper and lower sets. The lower lymph nodes are dissected to the inner edge of the smaller pectoral muscle, thus including both the level I and level II elements (Fig. 1).

The 28 patients showed an age range of $36-75$ years (mean : 60.1 years), with clinical staging showing Stage 0 in 7 cases, Stage I in 14 cases and Stage II in 7 cases.

\section{(STUDY 2)}

Between April of 2000 and January of 2001, 58 patients underwent surgery for primary breast cancer accompanied by axillary lymph node dissection in Higashi Tokushima National Hospital. After exclusion of four patients in whom lower axillary dissection was not carried out because of an abnormal course of the second intercostobrachial nerve, 54 patients were included in this study.

Following the usual procedure for level I and II node dissection, first lower axillary dissection is carried out, and then the level I \& II lymph nodes remaining in the upper set are dissected. Thus, level I and II lymph nodes are completely dissected. Then the results of examination of the lymph nodes removed by the lower axillary dissection and those removed by the level I and II node dissection were compared.

The 54 patients showed an age range of 30-72 years (mean: 50.5 years), with clinical staging showing Stage I in 19 cases, Stage II in 31 cases and Stage III in 4 cases.

Clinical pathological staging was performed in accordance with the TNM Classification of Malignant Tumours (Fifth Edition) published by the UICC. This method was carried out under enough informed consent to the patient made the target. 


\section{RESULTS}

(STUDY 1)

The classification of dissected axillary lymph nodes in the lower axillary dissection showed a range of 2-14 (mean : 7.6). An observation period after the operation was 50-100 months (mean : 60.8 months). Complications were the pain of the operated arm ( 3 cases), pooling of effusion to axiller ( 1 case), and axillary lymphnodes recurrence (1 case). Swelling of the operated arm wasn't seen (Table 1).

Table 1. Complications of lower axillary dissection

\begin{tabular}{ll}
\hline Pain of the operatid arm & 3 \\
Pooling of effusion to axiller & 1 \\
Swelling of the operated arm & 0 \\
Axillary lymphnodes recurrence & 1 \\
\hline
\end{tabular}

An observation period after the operation was 50-100 months (mean : 60.8 months).

\section{(STUDY 2)}

The classification of dissected axillary lymph nodes in the lower axillary dissection showed a range of 3-13 (mean : 6.5), while in the level I and II node dissection the range was 6-23 nodes (mean : 14.8).

Comparison of Pathological Classification of Lymph Node Metastases (pN) in Lower Axillary Dissection and Level I and II Node Dissection (Table 2)

Both the lower axillary dissection and the level I and II node dissection revealed 32 cases to be $\mathrm{pN} 0$. In addition, there were no cases diagnosed as $\mathrm{pN} 0$ on the

Table 2. Status of Metastasis-Positive Lymph Nodes (All Cases)

\begin{tabular}{cccc}
\hline & Low & $\begin{array}{c}\text { Level } \\
\text { I and II }\end{array}$ & $\begin{array}{c}\text { No. of } \\
\text { patients }\end{array}$ \\
\hline (Diagnostic accuracy rate 94\%) & 0 & 0 & 32 \\
& $1-3$ & $1-3$ & 0 \\
$1-3$ & $>3$ & 3 \\
$>3$ & $>3$ & 7 \\
total & & 54
\end{tabular}

The status of metastasis-positive lymph nodes $(\mathrm{pN})$, determined by postoperative histopathological studies, was compared between the lower axillary dissection and level I and II dissection methods. The absence of positive nodes was classified as $\mathrm{pN} 0$, whereas 1-3 positive nodes were classified as pN1bi and 4 or more $(>3)$ positive nodes were classified as pN1bii. The diagnostic accuracy rate (94\%) shows the percentage of cases for which the findings were the same with both the lower axillary dissection and level I and II dissection methods.
Table 3. Sensitivity and specificity of lower axillary dissection (All Cases)

\begin{tabular}{cccc}
\hline & pN0 & pN1bi & pN1bii \\
\hline Sensitivity & 100 & 100 & 70 \\
Specificity & 100 & 100 & 100 \\
\hline
\end{tabular}

basis of the lower axillary dissection but positive for metastasis based on the level I and II node dissection. On the other hand, three cases were diagnosed as pN1bi on the basis of the lower axillary dissection but pN1bii in the level I and II node dissection. For the total 54 cases, the rate of agreement for $\mathrm{pN}$ between the lower axillary dissection and the level I and II node dissection was $94 \%$. The sensitivity and specificity of lower axillary dissection were each $100 \%$ with the pN0 case and the pN1bi case, specificity was $100 \%$ and sensitivity was $70 \%$ with the pN1bii case (Table 3 ).

Comparison of Pathological Classification of Lymph Node Metastases ( $p N)$ in Lower Axillary Dissection and Level I and II Node Dissection as function of preoperative disease stage (Table 4)

Nineteen cases were classified as Stage I, and the rate of agreement for $\mathrm{pN}$ between the lower axillary

Table 4. Status of Metastasis-Positive Lymph Nodes (as Function of Preoperative Disease Stage)

\begin{tabular}{cccc}
\hline & Low & $\begin{array}{c}\text { Level } \\
\text { I \& II }\end{array}$ & $\begin{array}{c}\text { No. of } \\
\text { patients }\end{array}$ \\
\hline Stage I & 0 & 0 & 16 \\
(Diagnostic accuracy rate 100\%) & 0 & $1-3$ & 3 \\
& total & & 19 \\
\hline Stage II & 0 & 0 & 16 \\
(Diagnostic accuracy rate 90\%) & $1-3$ & $1-3$ & 8 \\
& $1-3$ & $>3$ & 3 \\
& $>3$ & $>3$ & 4 \\
& total & & 31 \\
\hline
\end{tabular}

The cases were stratified as a function of their preoperative disease stage (Stage I or Stage II), and comparison of the number of metastasis-positive lymph nodes (pN) found by the lower axillary dissection and level I and II dissection methods was performed as described in Table 1 .

Table 5. Sensitivity and specificity of lower axillary dissection (as Function of Preoperative Disease Stage)

\begin{tabular}{ccccc}
\hline & & $\mathrm{pN} 0$ & $\mathrm{pN} 1 \mathrm{bi}$ & $\mathrm{pN} 1 \mathrm{bii}$ \\
\hline Stage I & Sensitivity & 100 & 100 & - \\
& Specificity & 100 & 100 & - \\
\hline \multirow{2}{*}{ Stage II } & Sensitivity & 100 & 100 & 57 \\
& Specificity & 100 & 100 & 100 \\
\hline
\end{tabular}


dissection and the level I and II node dissection was $100 \%$. The sensitivity and specificity of lower axillary dissection were $100 \%$ each (Table 5).

Thirty-one cases had Stage II disease. Both the lower axillary dissection and the level I and II node dissection indicated 16 of those cases to be pN0, eight cases to be pN1bi and four cases to be pN1bii. On the other hand, three cases were evaluated as p1Nbi by the lower axillary dissection but pN1bii by the level I and II node dissection. Accordingly, the lower axillary dissection and the level I and II node dissection showed a $90 \%$ rate of agreement for the pN status of the 31 Stage II cases. The sensitivity and specificity of lower axillary dissection were each $100 \%$ with the pN0 case and the pN1bi case, specificity was $100 \%$ and sensitivity was $57 \%$ with the pN1bii case (Table 5).

Comparison of Pathological Classification of Lymph Node Metastases ( $p N)$ in Lower Axillary Dissection and Level I and II Node Dissection as function of preoperative lymph nodes status (Table 6)

Thirty of the patients were clinically diagnosed as being free of axillary lymph node metastases. Seven of these patients were found to be histologically positive for lymph node metastases, but the lower axillary dis-

Table 6. Status of Metastasis-Positive Lymph Nodes (as Function of Preoperative Lymph Node Status)

\begin{tabular}{cccc}
\hline & Low & $\begin{array}{c}\text { Level } \\
\text { I and II }\end{array}$ & $\begin{array}{c}\text { No. of } \\
\text { patients }\end{array}$ \\
\hline N0 & 0 & 0 & 23 \\
(Diagnostic accuracy rate 100\%) & $1-3$ & $1-3$ & 7 \\
& total & & 30 \\
\hline N1 & 0 & 0 & 9 \\
(Diagnostic accuracy rate 88\%) & $1-3$ & $1-3$ & 5 \\
& $1-3$ & $>3$ & 3 \\
& $>3$ & $>3$ & 7 \\
& total & & 24 \\
\hline
\end{tabular}

The cases were stratified as a function of their preoperative lymph node metastasis status (N0 or N1), and comparison of the number of metastasis-positive lymph nodes $(\mathrm{pN})$ found by the lower axillary dissection and level I and II dissection methods was performed as in Table 1.

Table 7. Sensitivity and specificity of lower axillary dissection (as Function of Preoperative Lymph Node Status)

\begin{tabular}{ccccc}
\hline & & pN0 & pN1bi & pN1bii \\
\hline \multirow{2}{*}{ N0 } & Sensitivity & 100 & 100 & - \\
& Specificity & 100 & 100 & - \\
\hline \multirow{2}{*}{ N1 } & Sensitivity & 100 & 100 & 70 \\
& Specificity & 100 & 100 & 100 \\
\hline
\end{tabular}

section and the level I and II node dissection showed a $100 \%$ rate of agreement for the $\mathrm{pN}$ status of these 30 cases. The sensitivity and specificity of lower axillary dissection were $100 \%$ each (Table 7).

Twenty-four of the patients had been clinically diagnosed as positive for axillary lymph node metastases prior to the operation. Histologically, nine of those patients were found to be free of axillary lymph node metastases, and the degree of agreement between the lower axillary dissection and the level I and II node dissection on the $\mathrm{pN}$ status of these cases was $100 \%$. In three patients, the number of metastatic axillary lymph nodes was 3 or less according to the lower axillary dissection but 4 or more according to the level I and II node dissection. Therefore, for these cases clinically diagnosed as having lymph node metastases (N1), the agreement rate between the lower axillary dissection and the level I and II node dissection on the $\mathrm{pN}$ status was $88 \%$. The sensitivity and specificity of lower axillary dissection were each $100 \%$ with the pN0 case and the pN1bi case, specificity was $100 \%$ and sensitivity was $70 \%$ with the pN1bii case (Table 7).

\section{DISCUSSION}

Following the 1977 report by Fisher et al. (2) of their randomized clinical trial, Donegan (3) reported that axillary lymph node dissection did not contribute to improving the survival results in breast cancer patients. Since then, the objectives of axillary lymph node dissection have been as an aide in estimation of the prognosis and for limiting local axillary recurrence. In addition, it has been reported that there are no differences in the survival rate between standard radical mastectomy and modified radical mastectomy $(3,4)$. Moreover, in 1991, NIH Consensus Conference (5) released a recommendation that the standard treatment for breast cancer should be simple mastectomy plus level I and II axillary lymph node dissection. However, level I and II axillary lymph node dissection causes various complications, such as swelling of the arm on the dissected side, pain, etc. Other problems exist, including: skip metastases to level III in 3\% of patients (6), and these cannot be diagnosed by level I and II axillary lymph node dissection ; patients who are positive for metastases at levels I and II are often found to have metastasis to level III (6), but these are not dissected and can result in under-staging. Accordingly, sentinel lymph node biopsy is currently undergoing clinical trials as a new technique at various institutions.

The principal advantages of sentinel lymph node 
Table 8. Three Cases of Under-Staging

\begin{tabular}{ccccccccc}
\hline \multicolumn{3}{c}{ Preoperative Status } & \multicolumn{4}{c}{ Lymphnode Metastasis Status } \\
\hline $\mathrm{T}$ & $\mathrm{N}$ & Stage & Low $(+)$ & $/$ & Low(all) & $\begin{array}{c}\text { Level } \\
\text { I and II(+) }\end{array}$ & $/$ & $\begin{array}{c}\text { Level } \\
\text { I and II(all) }\end{array}$ \\
\hline 2 & 1 & II B & 1 & $/$ & 3 & 8 & $/$ & 18 \\
2 & 1 & II B & 3 & $/$ & 4 & 4 & $/$ & 10 \\
2 & 1 & II B & 3 & $/$ & 3 & 4 & $/$ & 13 \\
\hline
\end{tabular}

Three cases were unders-staged by the lower axillary dissection method compared with the level I and II dissection method. None of these cases had preoperative distant metastases (M0).

biopsy are as follows. It reduces postoperative complications because it makes it possible to avoid unnecessary axillary lymph node dissection in patients who are found to be negative for axillary lymph node metastasis. It also enables diagnosis of skip metastases. Third, this technique makes it possible to achieve accurate staging of node-positive breast cancer patients because it involves dissection to level III. Finally, sentinel lymph node biopsy makes it possible to control local recurrence. By virtue of these attributes, it is anticipated that sentinel lymph node biopsy will become the standard technique in the future. A 75\% diagnostic accuracy rate was reported for sentinel lymph node biopsy when the dye method was used alone (7). Combined use of the RI method is becoming common with the objective of elevating the accuracy rate, and a rate of $93 \%$ was reported (7). However, combination of sentinel lymph node biopsy and RI can only be carried out at institutions that are equipped to handle these hazardous materials, which makes it difficult for this combined technique to be adopted in ordinary hospitals.

It was in consideration of this background situation that we conceived of the lower axillary dissection approach for the management of breast cancer patients. Our concept is an adaptation of two earlier-reported methods : pectoral node biopsy, proposed by Forrest et al ., in 1982 (8), and lower axillary sampling, reported by Steele et al., in 1985 (9). That is, our technique entails dissection, not sampling. At the time of those earlier reports, it was thought that sampling was sufficient for estimating the prognosis, but a subsequent report stated that at least 10 lymph nodes had to be examined in order to make an accurate estimate of the prognosis (10). As a result, those earlier methods did no become standard approaches. In our present technique of lower axillary dissection, the objectives of carrying out dissection of the lower axillary lymph nodes rather than mere sampling are to increase the number of resected lymph nodes and to conserve the main lymph canals, which are known from experience to run from the upper arm cephalic to the intercostobrachial nerve. In fact, prior to the present study, we carried out a comparison of the postoperative complications after lower axillary dissection and after level I and II dissection.

That comparison revealed that the incidence of postoperative swelling of the operated arm showed a range of 2.7-5.0\% after level I \& II dissection versus a range of $0-2.8 \%$ after lower axillary dissection. Postoperative swelling didn't happen to every patient by STUDY 1 . And, the place where it recurred is level III with one person who brought about lymph node recurrence. As for the side effect which is characteristic of lower axillary dissection, it doesn't think easily.

In our present study of 54 breast cancer patients in whom lower axillary dissection was able to be performed, the number of axillary lymph nodes dissected showed a range of 3-13(mean : 6.5) in the lower axillary dissection and a range of 6-23(mean:14.8) in the level I and II dissection. The diagnostic accuracy rate for the total 54 patients was $94 \%$, which is superior to the $90 \%$ reported for the sampling method (8). In addition, in the case of sentinel lymph node biopsy, the rate of detection of the sentinel lymph node was $75 \%$ when using the dye method alone, whereas it was $93 \%$ when combined with use of an RI (7). Thus, the lower axillary dissection approach can be considered to be more accurate. Furthermore, it has been reported that the false-positive rate with sentinel lymph node biopsy is $4.5-5.6 \%$ (7), and in consideration of this, as well, it can be thought that our $94 \%$ diagnostic accuracy rate by means of lower axillary dissection compares favorably with the results obtained by sentinel lymph node biopsy. Recently, there is a report that five node biopsy from the surroundings of the sentinel lymph node in the early breast cancer was effective (11). In particular, our present study showed $100 \%$ accuracy rate, sensitivity, and specificity in the diagnosis of clinical Stage I breast cancer cases and cases with a clinical classification of N0. Moreover, the three cases which were under-staged (Table 8) in this study were each Stage IIB cases showing T2N1M0. These findings also indicate the potential of lower axillary dissection to replace level I and II dissection, es- 
pecially in Stage 0 and Stage I cases of breast cancer.

Regarding the procedures to be applied after performing lower axillary dissection, for cases found to be node-positive by frozen sample diagnosis during the operation, complete dissection should be performed to level III. For node-negative cases, the subsequent procedures are the same as for cases found to be nodenegative by level I and II dissection. It will be best to perform follow-up observation of the axillary region by ultrasound examinations.

Thus, lower axillary dissection is seen to have many advantages, such as that it does not require any special facilities or equipment, it results in few complications and it affords a high diagnostic accuracy rate. However, it must be noted that some problems remain with the lower axillary dissection approach. For example, the number of lymph nodes to be dissected can vary between individual patients as a result of differences in the location of the delineating intercostobrachial nerve, while the technique does not permit diagnosis of skip metastases.

It is hoped that the future will bring about a more thorough understanding of the mammary gland lymph flow, and that it will be possible to establish a dissection method which is even safer, more broadly applicable and causes fewer complications than the currently available techniques.

\section{ACKNOWLEDGEMENTS}

We thank Dr. Yuji Takahashi, Second Department of Surgery, The University of Tokushima Faculty of Medicine, for his help in preparing this manuscript. We also thank Prof. Tadaoki Morimoto, School of Health Sciences, The University of Tokushima, for his critical review of this manuscript.

\section{REFERENCES}

1. Sobin LH, Wittekind C : "TNM Classification of Malignant Tumours (Fifth Edition).” International Union Against Cancer, 1977

2. Fisher B, Montague E, Redmond C, Barton B, Borland D, Fisher ER, Deutsch M, Schwarz G,
Margolese R, Donegan W, Volk H, Konvolinka C, Gardner B, Cohn IJ, Lesnick G, Cruz AB, Lawrence W, Nealon T, Butcher H, Lawton R: Comparison of radical mastectomy with alternative treatments for primary breast cancer; A first report of results from a prospective randomized clinical trial. Cancer 39 : 2827-2839, 1977

3. Donegan WI: Surgical clinical trial. Cancer 53: 691-699, 1984

4. Fisher B, Redmond C, Fisher ER, Bauer M, Wolmark N, Wickerham L, Deutsch M, Montague E, Margolese $\mathrm{R}$, Fostre $\mathrm{R}$ : Ten-year results of a randomized clinical trial conparing radical mastectomy and total mastectomy and total mastectomy with or without radiation. N Engl J Med 312 : 674-681, 1985

5. National Institute of Health Consensus Conference: Treatment of early stage breast cancer. JAMA $265:$ 391-395, 1991

6. Rosen PP, Lesser ML, Kinne DW, Beattie EJ : Discontinuous or "skip" metastases in breast carcinoma ;Analysis of 1228 axillary dissections. Ann Surg 197 : 276-283, 1983

7. O'Hea BJ, Hill AD, EI-Shirbiny AM, Yeh SD, Rosen PP, Coit DG, Borgen PI, Cody HS : Sentinel lymph node biopsy in breast cancer ; Initial experience at Memorial Sloan-Kettering Cancer Center. Am Coll Surg 186 : 423-427, 1998

8. Forrest APM, Stewart HJ, Roberts MM, Steele MB :Simple mastectomy and axillary node sampling (pectoral node biopsy) in the management of primary breast cancer. Ann Surg1 $96: 371-378$, 1982

9. Steele RJC, Forrest APM, Gibson T, Stewart HJ, Chetty $\mathrm{U}$ : The efficacy of lower axillary sampling in obtaining lymph node status in breast cancer; A controlled randomized trial. Br J Surg 72 : 368-369, 1985

10. Kiricuta CI, Tausch J:A mathematical model of axillary lymph node involvement based on 1446 complete axillary dissections in patients with breast carcinoma. Cancer 69 : 2496-2501, 1992

11. Ahlgren J, Holmberg L, Bergh J, Liljegren G : Fivenode biopsy of the axilla : an alternative to axillary dissection of levels I-II in operable breast cancer. Eur J Surg Oncol 28 : 97-102, 2002 